\title{
Comparação de modelos de previsão voltados à manutenção preditiva na indústria automobilística a partir de dados de inspeção de qualidade
}

\author{
Guinther K. da Costa*. Ricardo M. Kagami**. Ricardo A. Diogo*. Neri dos Santos***. \\ Roberto Z. Freire** \\ *Departamento de Engenharia de Controle e Automação \\ ** Programa de Pós-Graduação em Engenharia de Produção e Sistemas (PPGEPS) \\ Pontifícia Universidade Católica do Paraná (PUCPR), Rua Imaculada Conceição, 1155, 80215-901, Curitiba, Brasil \\ (e-mails: guintherkovalski@gmail.com, ricardo.zanotto@pucpr.br, ricardo.diogo@pucpr.br, roberto.freire@pucpr.br). \\ *** Programa de Pós-graduação em Engenharia e Gestão do Conhecimento (PPG-EGC) \\ Universidade Federal de Santa Catarina (UFSC), R. Eng. Agronômico Andrei Cristian Ferreira, s/n, 88040-900, \\ Florianópolis, Brasil (e-mail: neri@egc.ufsc.br).
}

\begin{abstract}
Predictive maintenance can be used for the optimization of machine availability, reducing maintenance costs, improving quality management and decision-making. This paper proposes the use of 5 time-series forecasting methods, being two classical approaches: Exponential Smoothing and Autoregressive Integrated Moving Average (ARIMA); and three based on machine learning: Radial Basis Function Neural Networks (RBF-NN), Support Vector Regression (SVR), and Long-Short Term Memory (LSTM). The meta-parameters of the models were optimized using Differential Evolution (DE) algorithm. In the presented analysis, a quality inspection database from an automaker factory floor was presented. The main idea was to predict deviations from tolerance, anticipating corrective actions in the production process. As a result, it was possible to compare the efficiency of the 5 models in relation to three time series analyzing their characteristics, strengths and drawbacks.
\end{abstract}

Resumo: A manutenção preditiva pode ser usada na otimização da disponibilidade de máquinas, redução de custos de manutenção, gestão da qualidade e processo de tomada de decisões. Neste trabalho realizouse o estudo de 5 modelos de previsão de séries temporais, dois clássicos: Suavização Exponencial e Autorregressivo Integrado de Médias Móveis (ARIMA), e três baseados em aprendizado de máquina: Rede Neural de Base Radial (RBF-NN), Regressão por Vetores de Suporte (SVR) e Memória de Curto e Longo Prazo (LSTM). Os meta-parâmetros dos modelos foram otimizados usando o algoritmo de Evolução Diferencial (DE). A base de dados utilizada neste estudo é proveniente da inspeção da qualidade no chão de fábrica de uma montadora. Neste contexto, a ideia principal foi prever desvios relacionados à tolerância, antecipando ações de correção no processo produtivo. Como resultado, foi possível comparar a eficiência dos 5 modelos em relação a três séries temporais analisando suas características, vantagens e desvantagens.

Keywords: Predictive maintenance, quality inspection, prediction models, machine learning, differential evolution.

Palavras-chaves: Manutenção preditiva, inspeção de qualidade, modelos de previsão, aprendizado de máquina, evolução diferencial.

\section{INTRODUÇÃO}

Em um cenário de mercado competitivo, um fator importante para companhias envolvidas no desenvolvimento de produtos é a capacidade de reduzir o tempo de produção, considerando custos reduzidos e qualidade adequada (Colosimo \& Senin, 2011). A manutenção de ativos também cumpre um importante papel neste contexto. A manutenção preditiva requer o monitoramento contínuo do equipamento para detectar e diagnosticar defeitos. Assim, apenas quando um defeito é detectado, o plano de manutenção será executado (Levitt, 2011).

Na indústria, o monitoramento e obtenção de dados leva a um novo paradigma, onde os dados também contribuem para o capital de uma companhia. Na era dos objetos conectados, da facilidade na coleta de dados e do acesso remoto instantâneo, a exploração dos dados se torna uma ferramenta valiosa, servindo de base para a tomada de decisão (Chebel-Morello, Nicod \& Varnier, 2017).

Os modelos de previsão de séries temporais constituem um conjunto de ferramentas eficazes para exploração de dados, sendo esta uma área de pesquisa dinâmica que vem atraindo a atenção não só da comunidade científica, mas de médias e grandes empresas, onde o objetivo principal é coletar e estudar observações passadas de um determinado processo para desenvolver um modelo capaz de descrever seu comportamento no futuro. Existe uma ampla variedade de métodos, desde os clássicos (Gooijera \& Hyndman, 2006), até os mais recentes baseados em aprendizado de máquina e aprendizado profundo (Gamboa, 2017). 
Em trabalhos anteriores com aplicação semelhante à proposta neste estudo, voltada ao setor automotivo, pode-se citar o trabalho apresentado por Juster et al. (2001), onde foram feitas previsões de qualidade estética de espaço e desnível a partir de simulações numéricas em modelos 3D. Já Krugh et al. (2016) apresentaram previsões de propensão a defeitos em montagens manuais automotivas de conexões elétricas, usando um modelo de regressão que tem, entre seus preditores, variáveis do processo como o tempo e a complexidade da operação, além das séries temporais de defeitos por unidade. Por fim, no trabalho de Ahmed \& Kim (2018) foram feitas previsões de qualidade de solda na indústria automotiva, usando árvores de decisão.

Neste contexto, este trabalho objetiva avaliar 5 modelos de previsão de séries temporais aplicados à precisão de medições e condições da qualidade de produtos. Atrelado a esta análise, pretende-se melhorar o tempo de reação a não conformidades do processo produtivo. Os 5 modelos foram treinados, validados e seus meta-parâmetros otimizados através de um algoritmo de Evolução Diferencial. Como resultados, apresenta-se ao final deste artigo uma comparação entre o desempenho dos métodos, bem como uma relação entre as características de cada série temporal analisada.

Esse trabalho está dividido em 5 partes. Na seção 2 apresentam-se o problema e a base de dados, na seção 3 apresenta-se a fundamentação teórica dos modelos utilizados, na seção 4 são explicados os procedimentos de treino e validação. Por fim, a seção 5 expõe as conclusões e possibilidades de trabalhos futuros associadas a esta pesquisa.

\section{DESCRIÇÃO DO PROBLEMA}

$\mathrm{Na}$ indústria automobilística, o controle de qualidade tem um papel importante, servindo a gestão dos meios produtivos com informações que levam à reatividade dos processos, onde um dos principais aspectos a serem mensurados é aquele relacionado à qualidade percebida pelo cliente. Um caso de controle de qualidade, com considerável impacto sobre a qualidade percebida pelo cliente final, diz respeito aos espaços e desníveis na lataria de um veículo, ilustrado na Fig. 1.

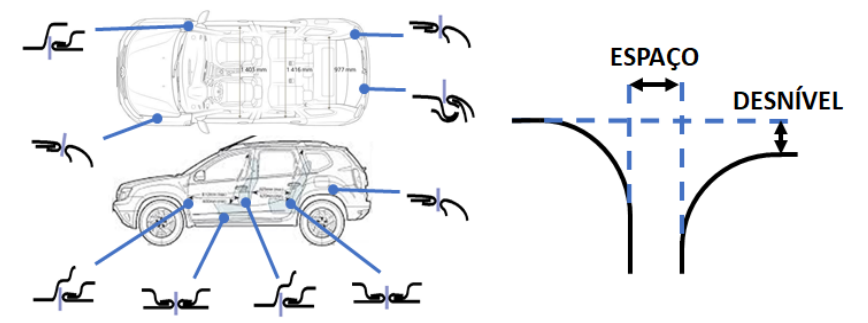

Fig. 1 Espaços e desníveis.

A base de dados utilizada neste trabalho consiste nas medições dos espaços e desníveis feitas na etapa de inspeção final de uma linha de produção.

\subsection{Base de dados}

A base de dados consiste em medições compiladas semanalmente, constituídas por 167 medições, em 113 pontos.
Por se tratar de um volume grande de dados, com a finalidade de limitação de escopo, foram selecionadas as 3 variáveis com mais valores fora dos limites de tolerância.

A fim de manter o sigilo dos dados, estes foram normalizados ao intervalo $[0,1]$, onde os valores das tolerâncias seguiram a mesma transformação linear.

\section{MODELOS DE PREVISÃO DE SÉRIES TEMPORAIS}

Esta seção descreve os 5 modelos de previsão de séries temporais adotados neste trabalho, são eles: Suavização Exponencial, Modelo Autorregressivo Integrado de Médias Móveis (ARIMA), Regressão por Vetores de Suporte (SVR), Rede Neural de Base Radial (RBF-NN) e Rede Neural de Memória de Curto e Longo Prazo (LSTM).

\subsection{Suavização Exponencial}

Um modelo de suavização exponencial (Goodell \& Holt, 1956) pode ser simples, aditivo ou multiplicativo. No método de Suavização Exponencial Multiplicativo o nível médio, a tendência e a sazonalidade da série são estimados respectivamente por $L[t], T[t]$ e $S[t]$, conforme o modelo expresso nas equações descritas em (1):

$$
\begin{gathered}
L[t]=\alpha \frac{y[t]}{S[t-h]}+(1-\alpha)(L[t-1]+T[t-1]), \\
T[t]=\beta(L[t]-L[t-1])+(1-\beta) T[t-1], \\
S[t]=\gamma \frac{y[t]}{L[t]}+(1-\gamma) S[t-s],
\end{gathered}
$$

tal que $0<\alpha, \beta, \gamma<1$, onde $\alpha, \beta$ e $\gamma$ são fatores suavizadores de nível, tendência e sazonalidade, respectivamente. O termo $s$ é o período usado para a janela de observações passadas referente a entrada do modelo, sendo as previsões dadas por:

$$
\hat{\mathrm{y}}[t]=L[t]+t T[t]+S[t-s+h] .
$$

\subsection{Modelo Autorregressivo Integrado de Médias Móveis}

Um modelo de média móvel integrada auto regressivo (ARIMA) (Box \& Jenkins, 1970), na sua forma sazonal, tem a notação $\operatorname{ARIMA}(p, q, d)(P, Q, D) s$, onde $p$ é a ordem (tamanho da janela de observações) do modelo, $d$ é a ordem de diferenciação e $q$ é a ordem do processo de média móvel. Os parâmetros $P, Q, D$ tem respectivamente o mesmo significado, mas para o componente sazonal. Assim, a saída prevista pode ser descrita como:

$$
\hat{\mathrm{y}}[t]=\mu+\frac{\theta(B) \theta_{s}\left(B^{s}\right)}{\emptyset(B) \emptyset_{s}\left(B^{s}\right)(1-B)^{d}\left(1-B^{s}\right)^{D}} Z
$$

onde $\emptyset_{s}\left(B^{s}\right)$ é o operador auto-regressivo e $\theta_{s}\left(B^{s}\right)$ é o operador sazonal da média móvel, ambos com representação polinomial. Por fim, $Z$ é um processo puramente aleatório normalmente distribuído de média zero e variância $\sigma_{Z}^{2}$. 


\subsection{Rede Neural de Função de Base Radial}

A rede neural de base radial (RBF-NN) (Broomhead \& Lowe, 1988), é uma rede neural com 3 camadas, sendo uma de entrada, uma oculta e a última de saída.

Cada neurônio na camada oculta tem uma função de base radial (RBF) $\emptyset(r)$ não linear. $\emptyset_{0}(x)=1$ corresponde ao bias na camada de saída, enquanto $\emptyset_{i}(x)=\left(x_{i}-c_{i}\right)$, onde $c_{i}$ é o centro do $i$-ésimo neurônio. A camada oculta faz uma transformação não linear, e a camada de saída uma combinação linear que mapeia a não linearidade para um novo espaço. Desta forma, a rede RBF pode então alcançar uma solução global ajustando os pesos tal que o erro quadrático médio (MSE) seja minimizado usando um método de otimização linear.

Neste trabalho a RBF foi construída baseada na implementação feita por Arriaga (2018), com modificações na função de alocação dos $c_{i}$, onde utilizou-se K-means como método.

\subsection{Regressão por Vetores de Suporte (SVR)}

A regressão por vetores de suporte é baseada em Máquina de Vetores de Suporte (SVM) (Vapnik et al., 1997). Este modelo também consiste em uma rede neural de 3 camadas não paramétrica, já que depende do Kernel escolhido. Na regressão, o conjunto de dados de treinamento inclui variáveis preditoras e valores da resposta observados dado um conjunto de dados $\{x[t], y[t]\}$.

O objetivo é encontrar uma função $f(t)$ que se desvie de $y[t]$ por um valor não superior a uma margem $\varepsilon$ para todo ponto de treinamento $x[t]$. No caso de uma $f(t)$ linear:

$$
\forall t:|y[t]-(t \beta+b)|<\varepsilon
$$

É possível que nenhuma função $f(t)$ atenda a Eq. 5, neste caso, utiliza-se o conceito de variáveis de folga $\xi_{i}$ e $\xi_{i}{ }^{*}$ de maneira a atender a otimização. Assim,

$$
J(\beta)=\frac{1}{2} \beta^{\prime} \beta+C \sum_{i=1}^{N}\left(\xi_{i}+\xi_{i}{ }^{*}\right),
$$

\subsection{Rede Neural de Memória de Curto e Longo Prazo (LSTM)}

Redes neurais recorrentes (RNN) (Hopfield, 1982) são sistemas dinâmicos com representações dos estados temporais das entradas que introduzem o conceito de memória. No seu processo de treinamento, o desaparecimento dos gradientes surge como um problema, sendo um dos principais obstáculos relativos ao seu desempenho. Uma variação dos modelos de RNN chamado de Rede Neural de Memória de Curto e Longo Prazo (LSTM) foi proposta por Hochreiter e Schmidhuber (1997) como solução para o problema dos gradientes, esta foi adotada neste trabalho.

As RNN's conseguem, com tamanho significativamente menor quando comparadas a redes do tipo não recorrentes, ter precisão aproximada para problemas de regressão (Ke-Lin \& Swamy, 2013).

\subsection{Evolução Diferencial (DE)}

A evolução diferencial é um dos algoritmos da família de computação evolucionária bioinspirados que foi proposto por Storn \& Price (1997). Neste trabalho o algoritmo foi usado para otimizar os meta parâmetros de cada modelo de previsão de séries temporais descritos na seção anterior.

Durante a inicialização da DE, são definidos o tamanho da população $(N P)$, o espaço de busca e o número de gerações ou limiar de erro para a finalização do algoritmo. Nesta abordagem, a população inicial foi configurada com valores aleatórios para os parâmetros dentro dos limites préestabelecidos e o vetor alvo é tido como $x_{i, G}, i=1,2, \ldots, N P$, com dimensão NP. Para cada iteração, a estratégia de evolução do algoritmo gera novos vetores de parâmetros através da diferença ponderada entre dois vetores, com constante de mutação $F m$, somando o resultado a um terceiro vetor com índices aleatórios $r 1, r 2, r 3 \in\{1,2, \ldots, N P\}$, de acordo com a Eq. (6):

$$
v_{i, G+1}=x_{r 1, G}+F m\left(x_{r 2, G}-x_{r 3, G}\right) .
$$

\section{CONFIGURAÇÃO DOS MÉTODOS DE PREVISÃO}

Nesta seção descrevem-se os procedimentos de treinamento, validação, otimização e testes dos cinco modelos adotados neste estudo.

\subsection{Parâmetros dos Modelos}

Os parâmetros do algoritmo evolutivo foram padronizados para todos os modelos, utilizando-se de 10 indivíduos vezes o número de parâmetros, em 10 gerações, considerando $25 \%$ de recombinação e constante de mutação $F m$ de 0,5.

A Tabela 1 apresenta os parametros otimizados em cada um dos modelos apresentados das seções 3.1 a 3.5 e os seus respectivos espaços de busca. Já a tabela 2 descreve os demais parâmetros dos modelos.

\begin{tabular}{|c|c|c|c|}
\hline SE & $\begin{array}{c}\alpha \\
{[0,1]}\end{array}$ & $\begin{array}{c}\beta \\
{[0,1]}\end{array}$ & $\begin{array}{c}\gamma \\
{[0,1]}\end{array}$ \\
\hline ARIMA & $\begin{array}{c}p \\
{[0,3]}\end{array}$ & $\begin{array}{c}q, d, P, Q, \\
\quad D[0,2]\end{array}$ & - \\
\hline $\mathrm{RBF}$ & $\begin{array}{c}\sigma \\
{[0,1 ; 2]}\end{array}$ & $\begin{array}{c}\text { Neurônios } \\
{[70,150]} \\
\end{array}$ & - \\
\hline SVR & $\begin{array}{l}\text { Tipo de } \\
\Phi[0,4]\end{array}$ & $C[1 E 4,1 E 8]$ & $\begin{array}{c}\varepsilon \\
{[0,01 ; 0,5]}\end{array}$ \\
\hline LSTM & $\begin{array}{c}\text { Neurônios } \\
{[20,200]}\end{array}$ & $\begin{array}{l}\text { Momentum } \\
{[0,1 ; 0,99]}\end{array}$ & $\begin{array}{c}L R \\
{[0,01 ; 0,5]}\end{array}$ \\
\hline
\end{tabular}

Tabela 1. Parâmetros otimizados pela DE e espaços de busca 
Tabela 2. Parâmetros não otimizados e valores adotados

\begin{tabular}{|c|c|}
\hline RBF & $c_{i}$ : definidos por K-means \\
\hline SVR & Número máximo de iterações: $2 E 5$ \\
\hline LSTM & Camadas: $3 ;$ batch: $15 ;$ dropout: 0,$1 ;$ \\
& decay: $5 E-4$ \\
\hline
\end{tabular}

\subsection{Critérios de Avaliação de Desempenho}

Para avaliar a qualidade dos modelos obtidos, foram usados a raiz do erro médio quadrático (RMSE), o erro absoluto médio percentual simétrico (SMAPE), este devido a presença de zeros nos dados originais, o que impossibilitaria o uso da média percentual do erro absoluto (MAPE). Além dos dois critérios anteriormente citados, também foi adotado o ajuste do modelo (MF), que mede o quanto o modelo se ajusta aos dados reais.

$$
\begin{aligned}
R M S E & =\sqrt{\frac{1}{n} \sum_{t=1}^{N}(\mathrm{y}[t]-\hat{\mathrm{y}}[t])^{2},} \\
S M A P E & =\frac{100}{n} \sum_{t=1}^{N} \frac{|\hat{\mathrm{y}}[t]-\mathrm{y}[t]|}{\left(\frac{\mathrm{y}[t]+\hat{\mathrm{y}}[t]}{2}\right)}, \\
M F & =\frac{\sum_{t=1}^{N}(\mathrm{y}[t]-\hat{\mathrm{y}}[t])^{2}}{\sum_{t=1}^{N} \mathrm{y}[t]^{2}} 100 .
\end{aligned}
$$

Nas Eqs. (7) a (9), y[t] são os valores reais e $\hat{y}[t]$ os valores previstos pelos modelos.

A base de dados foi dividida seguindo a proporção de $81 \%$ e $19 \%$, ou 130 e 30 amostras respectivamente. De maneira que as 130 amostras foram divididas em 3 pacotes de 42 amostras onde $71 \%$ (30 amostras) eram destinadas a treino e 12 amostras (29\%) para validação. Este procedimento também é conhecido como validação cruzada aninhada. O RMSE médio nestas 3 validações foi definido como função de custo do algoritmo de evolução diferencial. Ao fim da otimização dos parâmetros, os modelos foram retreinados usando as 130 amostras de treino, com os parâmetros obtidos pela otimização, e então testados.

\section{RESULTADOS}

Analisando os dados das Figuras 2, 3 e 4 é possível observar que os modelos baseados em aprendizado de máquina obtiveram melhores ajustes aos dados seguindo a métrica do $\mathrm{MF}$, e que os modelos clássicos obtiveram melhor RMSE. Isto pode ter sido causado pela seleção dos parâmetros otimizáveis, como o tipo de $\Phi$ na SVM, ou a quantidade de neurônios na LSTM. Levando-se em conta a perspectiva da evolução diferencial, a mudança de um destes parâmetros descarta soluções passadas, uma vez que as suas variações podem levar a novos espaços de busca.

Por outro lado, a não otimização de parâmetros como a quantidade de camadas, dropout, ou o tamanho do batch de treinamento podem ter levado a RNN a convergir a mínimos locais. $\mathrm{O}$ mesmo pode ter ocorrido para $\mathrm{RBF}$, em relação à não otimização individual da largura e assimetria das funções de ativação.

A partir das previsões feitas pelos modelos gerados em 30 otimizações de meta-parâmetros com Evolução Diferencial, foi calculado um intervalo de confiança para a média com 29 graus de liberdade, $95 \%$ de confiança, utilizando distribuição $t$ de Student. Com isto obteve-se dois vetores com intervalos superior e inferior em relação a média das previsões. A região cinza se refere à área entre estes dois vetores, como pode ser observado nas Fig. 2, 3 e 4 .

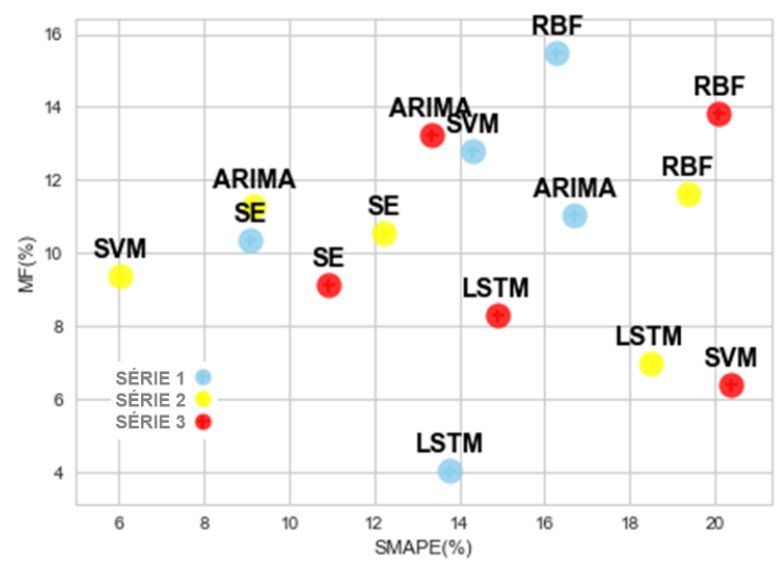

Fig. 2 Relação dos critérios MF e SMAPE.

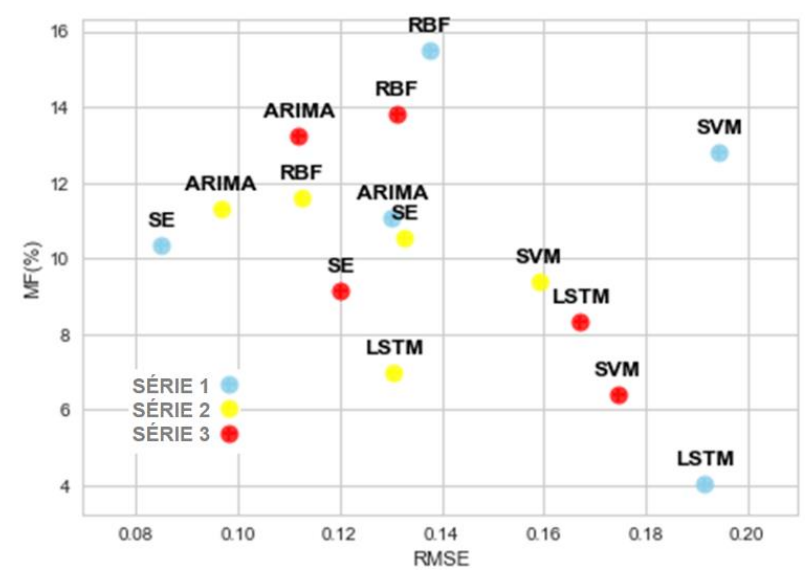

Fig. 3 Relação dos critérios MF e RMSE.

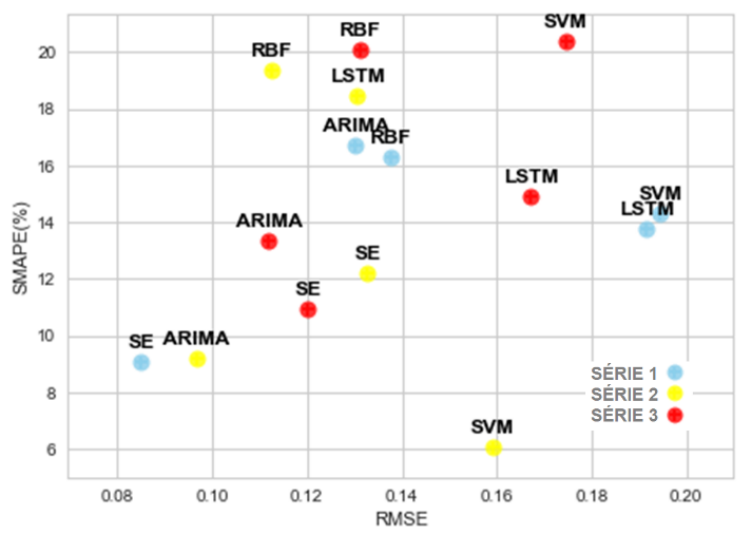

Fig. 4 Relação dos critérios SMAPE e RMSE. 
O modelo de suavização exponencial foi o que obteve o melhor RMSE, sobre a série 1, onde obteve-se uma boa previsão de tendência, mas com baixa precisão em relação aos picos sazonais. O modelo ARIMA, foi o que melhor respondeu à mudança abrupta presente na série 2. Já a RBF foi o modelo que melhor previu as oscilações com baixa amplitude e frequências relativamente mais altas, presentes na série 3.

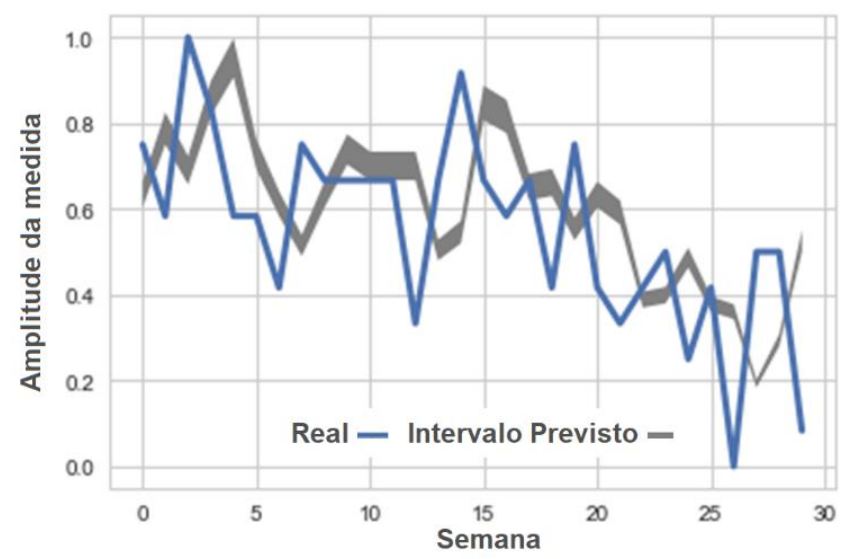

Fig. 5 Série 1 - Suavização Exponencial.

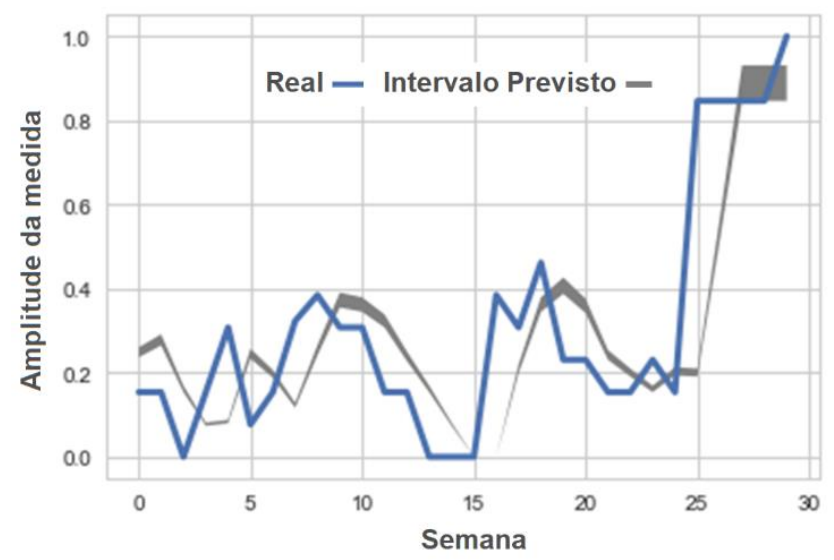

Fig. 6 Série 2- ARIMA.

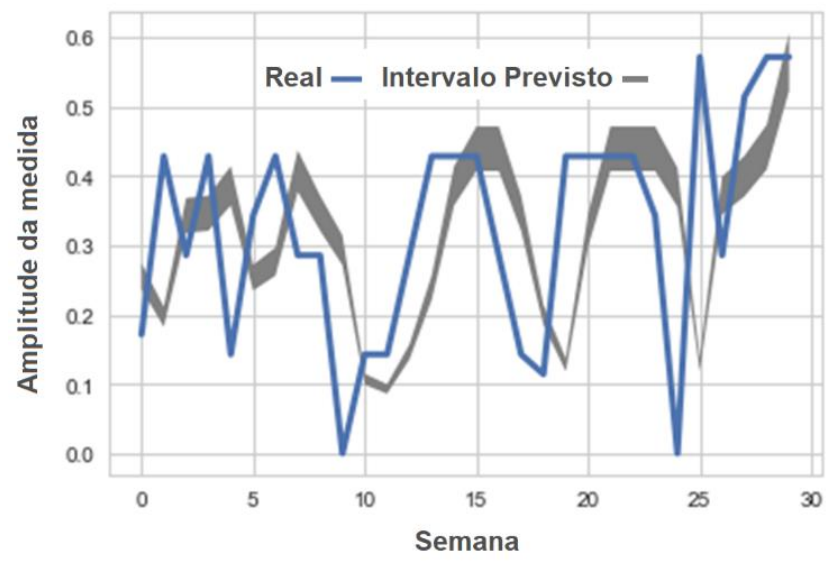

Fig. 7 Série $3-$ RBF.

Em relação ao processo de otimização, foi possível observar na Fig. 8 que a RBF obteve a menor variabilidade enquanto a suavização exponencial, apesar dos resultados com melhor RMSE médio, apresentou uma alta variabilidade.

Levando-se em conta os resultados obtidos, a título de seleção entre os três melhores modelos, foram realizados os testes de hipótese nula e alternativa, em relação ao RMSE:

$$
\begin{aligned}
& H_{0}: \mu(S E)=\mu(A R I M A)=\mu(S V M) \\
& H_{1}: \mu(S E) \neq \mu(\text { ARIMA) } \neq \mu(S V M)
\end{aligned}
$$

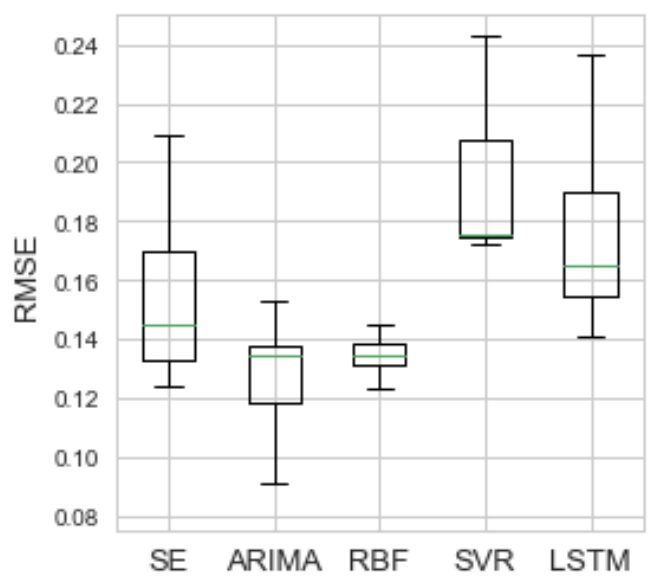

Fig. 8 RMSE na Validação.

Usando um teste de análise de variância (ANOVA), obteve-se $F=9,870$ e $p=1,36968 \mathrm{E}-4$, o que indica que existe diferença significativa entre as médias. Neste sentido, foi conduzido um teste de hipótese, onde:

$$
\begin{aligned}
& H_{0}: \mu(A R I M A)<\mu(R B F) \\
& H_{1}: \mu(A R I M A) \geq \mu(R B F)
\end{aligned}
$$

Utilizando se do teste $t$, obteve-se $t=2,161$ e $p=2,374 E-$ 2 , o que leva a rejeição da hipótese alternativa $H_{1}$, levando à conclusão estatística de que o modelo ARIMA tem menor RMSE médio, e portanto, representa um melhor modelo em relação a RBF para este caso de estudo. Procedimentos semelhantes para seleção de modelos de previsão podem ser encontrados em Permanasari et al (2010).

\section{CONCLUSÕES}

Esse estudo apresentou a implementação e comparação de 5 modelos de previsão de séries temporais, (Suavização Exponencial, ARIMA, RBF-NN, SVR, RNN-LSTM) onde os meta-parâmetros dos modelos foram otimizados por DE, para prever não conformidades a partir de uma base de dados composta por séries temporais de inspeção da qualidade coletados de chão de fábrica de uma montadora. Com o resultado foi possível comparar a eficiência dos 5 modelos em relação a três séries temporais analisando suas características, vantagens e desvantagens.

Para este estudo de previsão de séries temporais, baseadas em modelos de uma variável, os métodos clássicos superaram os métodos baseados em aprendizado de máquina. Resultados e conclusões semelhantes foram obtidos em (Makridakis, Spiliotis \& Assimakopoulos, 2018). Mesmo na ocasião de 
resultados similares, seguindo o princípio da parcimônia, considerando-se o custo computacional e a possibilidade de compreensão do que leva a cada previsão, os modelos clássicos foram as melhores alternativas considerando o conjunto de dados utilizado.

Por se tratar de séries com poucas amostras, em um processo com taxa de amostragem baixa, modelos baseados em aprendizado de máquina também têm grande desvantagem. Entretanto, estes modelos podem apresentar vantagens em relação aos métodos clássicos, no caso de presença de componentes não lineares ou fatores dificilmente modeláveis por abordagens estatísticas comuns.

Em trabalhos futuros, sugere-se melhorar a precisão das previsões utilizando-se de melhores técnicas de préprocessamento como a eliminação de tendência por diferenciação e séries dessazonalizadas, modelos multivariados e a combinação (multi-model ensemble) dos modelos de uma variável obtidos. O uso de dados de etapas anteriores no processo produtivo deve melhorar significativamente os resultados, mas trazem a necessidade de metodologias para seleção de preditores.

\section{AGRADECIMENTOS}

Os autores agradecem à Fundação Araucária do Estado do Paraná, Brasil, ao Conselho Nacional de Desenvolvimento Científico e Tecnológico (CNPq) do governo federal do Brasil sob a concessão 304783/2017-0, e a Pontifícia Universidade Católica do Paraná (PUCPR).

\section{REFERÊNCIAS}

Arriaga O., (2018). RBF-Network: Minimal implementation of a radial basis function network. GitHub repository: https://github.com/oarriaga/RBFNetwork/blob/master/R BFN.py

Brown, R.G. (1956). Exponential Smoothing for Predicting Demand. 10th National Meeting of the Operations Research Society of America, San Francisco.

Box, G., and Jenkins, G. (1970) Time Series Analysis: Forecasting and Control. Holden-Day, San Francisco.

Broomhead D. S., \& Lowe D. (1988) Multi variable Functional Interpolation and Adaptive Networks. Com plex Systems 2 (1988) 321- 355

Boser, B.E., Guyon, I., \& Vapnik, V.N. (1992). A training algorithm for optimal margin classifiers. InProceedings of the Fifth Annual Workshop of Computational Learning Theory, 5, 144-152. Pittsburgh, ACM.

Chebel-Morello, B., Nicod, J.-M., \& Varnier, C. (2017). From Prognostics and Health Systems Management to Predictive Maintenance 2. London: ISTE.

Colosimo, B. M., \& Senin, N. (2011). Geometric Tolerances. (B. M. Colosimo \& N. Senin, Eds.). London: Springer London

Drucker, H., Burges, C. J., Kaufman, L., Smola, A. J., \& Vapnik, V. (1997). Support vector regression machines. Advances in neural information processing systems.

Fernández-Delgado, M., Sirsat, M. S., Cernadas, E., Alawadi, S., Barro, S., \& Febrero-Bande, M. (2019). An extensive experimental survey of regression methods. Neural Networks, 111(December), 11-34.
Gooijer, J. G., Hyndman, R. J. (2006) 25 years of time series forecasting. International Journal of Forecasting 22(3), 443-473.

Hochreiter, S., \& Schmidhuber, J. (1997) Long Short-Term Memory. Neural Computation 9, 1997

Joel Levitt. (2011). Complete Guide to Preventive and Predictive Maintenance. Industrial Press Inc (2nd ed.).

John Gamboa (2017). Deep Learning for Time-Series Analysis. ArXiv Preprint ArXiv: 1701.01887

Juster, N. P., Fitchie, M. R., Taylor, S. J., Dew, P., Zhao, J., \& Maxfield, J. (2001). Visualising the impact of tolerances on cosmetic product quality. In International Conference on Engineering Design Iced (p. 8). GLASGOW.

Ke-Lin D., \& Swamy, M. N. S. (2013) Neural Networks and Statistical Learning ( Ke-Lin, D. \& M. N. S. Swamy)

Kim, K. Y., Ahmed, F. (2018) Semantic weldability prediction with RSW quality dataset and knowledge construction. Advanced Engineering Informatics Volume 38, October 2018

Krugh, M., Antani, Kavit., Mears, L., \& Schulte, J. (2016) Prediction of Defect Propensity for the Manual Assembly of Automotive Electrical Connectors. 44th Proceedings of the North American Manufacturing Volume 5, 2016

Makridakis, S., Spiliotis, E., \& Assimakopoulos, V (2018) Statistical and Machine Learning forecasting methods: Concerns and ways forward. PLOS ONE 13(3): e0194889

Permanasari A. E., Rambli D. R. A. , Dhanapal P. D. D. (2010). Forecasting Method Selection Using ANOVA and Duncan Multiple Range Tests on Time Series Dataset. IEEE International Symposium on Information Technology (January 2010), 10.1109

Sipos, R., Fradkin, D., Moerchen, F., \& Wang, Z. (2014). Logbased predictive maintenance, 1867-1876.

Storn, R., \& Price, K. (1997). Differential Evolution - A Simple and Efficient Heuristic for Global Optimization over Continuous Spaces. Journal of Global Optimization, 11(4), 341-359.

Susto, G. A., Wan, J., Pampuri, S., Zanon, M., Johnston, A. B., O'Hara, P. G., \& McLoone, S. (2014). An adaptive machine learning decision system for flexible predictive maintenance. IEEE International Conference on Automation Science and Engineering, 2014-(January 2016), 806-811.

Taylor, J. W., \& Jeon, J. (2018). Probabilistic forecasting of wave height for offshore wind turbine maintenance. European Journal of Operational Research, 267(3), 877890. 